stage, affording almost indisputable evidence of the method of infection, the infections were by the lung $\approx 6$, by the intestine 16 , by the ear 1 . Here the intesunal infections were to the others as 1 to 1.7. In the Luglish statistics the cases of tabes mesenterica in children five years and under are to the other forms of tuberculosis as 1 to 1.6. Dr. Still's observations are, therefore, a singular endorsement of the accuracy of the returns under the name of tabes mesenterica, and as the ages of the children covered by his autopsies were twelve years and under, he has really found a larger percentage of intestinal infection than is indicated by the statistics.

Dr. J. Walter Carr also referred to statistics of necropsies on tuberculous children at the Victoria Hospital." He found $\% 9$ in which the disease most probably started in the chest, and 20 in which it seemed to have begun in the abdomen. Here the relation between the two forms of infection is as 1 to 4 . In 26 children of early or limited tuberculosis, the thorax was alone affected in 12 cases, the abdomen in 7 , being in the proportion of 1 to 1.\%. Of 53 tuberculous children under two years of age the disease most probably began in the chest in 43 and in only 5 certainly in the abdomen, the proportion in this case being as 1 to 8.4 . Out of 27 children over five years of age, the disease began in the chest in 12 , in the abdomen in 6 , the relation being as 1 to 2 .

These statistics being all from English sources, are fairly comparable, and it appears to me they sustain Dr. Thorne's contention that the returns in England of tabes mesenterica represent with fair accuracy the abdominal tuberculosis of children.

Bollinger, in his address at the International Tuberculosis Congress of Berlin, in 1899, quoted with approval the record of autopsies by Heller (Kiel) of 248 tuberculous children which showed in 45.5 per cent. of the cases tuberculosis of the mesenteric glands. From these it was concluded that milk played a leading rôle in the so-called transmitted tuberculosis of children.

It is plain from what has been said, without quoting further statistics, that in some countries where bovine tuberculosis is very frequent, there is also great frequency of tuberculosis in children. Bollinger concludes that "although the tuberculosis of cattle and swine do not stand in the first line as source and starting-point of human tuberculosis, nevertheless-considering their enormous distribution and progressive additions, and the great danger from the ingestion of the milk of tuberculous cows-they are certainly for humanity the most important and most dangerous of all animal plagues and deserve the most earnest attention from the sanitarian and the state."

Admitting the subordinate rôle played by digestive infection in human tuberculosis, admitting that in England there is but one such case to nine cases of all forms of tuberculosis, still the matter is one deserving the most careful consideration of the sanitarian. But the harm does not stop with the cases directly infected by food, since these may in turn cause secondary infec. tions and thus give rise to a series of unlimited extent.

It is not my purpose to exaggerate in the least the danger from animal tuberculosis, but there has unfortunately been a tendency in recent years to make light of this danger. Let us take facts as our guide and not be unduly influenced by personal opinions. If it can be shown, as $I$ believe it has been, that animal

6. Brit. Med. Jour., Sept. 2, 1899, p. 626. tuberculosis may be communicated to man, then the presence of vast numbers of tuberculous animals in a country must be looked upon as a source of danger and one which it is our duty, as sanitarians and as citizens, to do our utmost to abolish.

\section{RELATIONS OF HYPERCHLORHYDRIA TO \\ "BILIOUS ATTACKS," SOME FORMS OF ECZEMA, GOUT, AND MUSCULAR RHEUMATISM. - PRELIMINARY REPORT.*}

GRAHAM CHAMBERS, B.A., M.B.

Professor of Dermatology, and Associate Professor of Clinical Medicine, Women's Medical College: Demonstrator of Clinical Medicine, University of Toronto; Physician to St. Michael's Hospital, etc. TORON'TO, ONTARIO.

Hyperacidity of the stomach is a common disturbance of secretion. The frequency of its occurrence is very difficult to determine, as there is no doubt that a moderate excessive secretion of hydrochloric acid may take place without producing subjective symptoms. I have on several occasions examined the gastric contents of patients with apparently normal digestion and found excess of hydrochloric acid, although in some of them there was a history of "bilious attacks," which were probably attacks of acute hyperacidity. It seems to me: therefore, that the gastric distress which is present in cases of hyperacidity is more or less due to the hyperesthesia of the mucous membrane of the stomach as well as to the excessive acid contents. This opinion is supported by the fact that in many cases of hyperchlorhydria pain comes on in a few minutes after the ingestion of food of any form. The commingling of these two neuroses-hyperchlorhydria and hyperesthesia gastrica-makes an investigation into the relations of the former to "bilious attacks," eczema, muscular rheumatism and gout a very difficult one, as I can not help but think that a general irritable condition of the gastric nerves must produce some changes in the sympathetic and cerebrospinal centers, wnich would no doubt lead or tend to lead to diseases in other organs. The investigation is also difficult because even if we find hyperchlorhydria associated with diseases of some other organ we have still to determine which was diseased primarily. or whether both pathologic conditions were not secondary to a disease in some other organ of the body, such as uremia, uratemia, nicotin poisoning, neurasthenia, etc.

My attention was first called to the subject about two years ago. I observed the internal treatment, hoth dietetic and medicinal, which $I$ was accustomed to use in cases of hyperchlorhydria, was approximately the same as that which I was using in some forms of acute eczema and in both cases it gave very satisfactory results. I then determined to investigate the relations of these two diseases and latterly I have extended the research to the whole subject-matter of my paper.

Before I give results of my observations, I wish to discuss and offer some suggestions as to how hyperchlorhydria may cause disease in other organs of the body. We know that the amount of blood in the portal system increases during the process of digestion. I think I am safe in stating that the more active the secretion of the stomach and intestines the greater the inflow of blood to the gastro-intestinal area. All the 
blood which enters the portal system must pass through the liver and hence the hyperemia of the stomach which occurs in hyperchlorhydria would tend to produce active congestion of the liver. 'The same pathologic condition might also be produced by the absorption of the toxic substances, the products of the disturbed digestıon, produced by the hyperchlorhydria. Thus we know that an excessive acid secretion interferes with the digestion of starch and does not interfere with at least some forms of fermentation. I have frequently observed considerable quantity of yeasts in gastric contents with normal hydrochloric acidity or even hyperacidity. I do not think that the secretion of hydrochloric acid bore any causal relation to the presence of yeast, as the growth of the latter no doubt resulted from the retention of food. If yeast can grow in the presence of $\mathrm{HCl}$, then it is probable that the latter will not have any deterrent action affecting the growth of some other forms of germs in presence of a suitable pabulum, and in all cases substances with variable degrees of toxicity would be produced and gain admittance to the portal circulation. Fermentation and putrefaction in the intestines are of frequent occurrence in cases of hyperchlorhydria and probahly result from the inflowing of the highly acid chyme containing large quantities of unchanged starch. The toxic substance thus produced would also be carried to the liver. We should also remember that these poisonous chemical bodies may not only cause active congestion of the liver but, changed or unchanged in constitution, pass on into the general circulation to produce disease in other organs. If active congestion of the liver results from the absorption of toxic substance from the stomach and intestines as well as from an excessive inflow of blood to the portal circulation, we have still to discuss the effects of pathologic hyperemia of the liver on the system in general. We know that the liver has varied and complex functions. It is at the same time a digestive, an excretory and assimilative organ, and ir would be quite natural for these functions to be disturbed by an excessive inflow of blood laden with toxic substances or even with an excess of food products. Defective metabolism might lead to an excess of urates in the blood and I believe that this is the case in hyperchlorhydria.

\section{HYPERCHLORHYDRIA AND "BILIOUS ATTACKS."}

When a patient complains of such subjective symptoms as headache, nausea, pain and discomfort in the region of the stomach, acid eructations, bitter taste in the mouth, disinclination to work. the diagnosis of bilious attacks is frequently made, particularly if the symptoms follow excessive eating or drinking. According to my experience, these attacks are very frequent in cases of chronic hyperchlorhydria, although they are not infrequent in patients who do not give a history of chronic indigestion. With the object of determining the activity of the secretion of $\mathrm{HCl}$ in this condition I produced emesis in a patient with the above symptoms one hour and a half after partaking of a breakfast of three pieces of toast and a cup of coffee and examined the romit. The total acidity and free $\mathrm{HCl}$ were 72 and 32 respectively, showing the presence of hyperchlorhydria. The patient had suffered from severe similar attacks previously, but they were of short duration, and in the intervals he had fairly good digestion. I think, therefore, that this was a case of acute hyperchlorhydria due to irritation of food which he had eaten a day or two previously, or an exacerbation of a mild form of hyperchlorhydria.

\section{RELATIONS OF HYPERCHLORHYDRIA AND SOME FORMS OF ECZEMA.}

That hyperacidity of the stomach bears some casual relation to some types of eczema I have no doubt. I am equally confident that there are cases of eczema occurring in persons with normal digestions. According to my experience symptoms of indigestion are of frequent occurrence in eczema, and are usually of the character that indicates hyperchlorhydria. In addition, I have examined the gastric contents of six cases of eczema with symptoms of dyspepsia; in five of these there was an excess of $\mathrm{HCl}$ in the gastric contents, the remaining case having normal acidity. Moreover, the internal treatment of acute irritable eczema which usually gives me the best results is about the same as that which I find most successful in cases of hyperchlorliydria.

I am unable to say in what manner the eczema is produced by the hyperchlorhydria, but I have some data which point to an excess of uric acid in with blood as the direct causative agent, and I have suggested in a previous part of my paper that the hyperchlorhydria may be the cause of the uratemia. However. I shall content myself for the present by reporting short clinical histories of some cases in practice which appear to indicate a relationship between these diseases.

CASE 1.-H. M., aged 42, market gardener, consulted me in the spring of 1900 on account of an eruption on his face, forearms, backs of hands, and fingers. His previous health had been fairly good, although he had suffered considerably from indigestion for two years before he came to me. He complained of heartburn, heaviness and slight pain after eating. His tongue was heavily coated and his bowels were constipated. The appetite was fairly good. The eruption on account of which he sought advice began two weeks previously on the backs of the hands; it then extended to the fingers and forearms, and lastly to the face. The rash had all the characters of acute vesicular eczema. I gave the patient a test breakfast and an analysis of the gastric contents revealed the presence of hyperchlorhydria. The patient was treated as follows: a mixture of black wash and calamin lotion was applied to the eczematous patches and the diet and internal medicine were the same as those indicated in hyperclilorhydria. Under this treatment he made a rapid and complete recovery from both the eczema and indigestion.

CASE 2.-A. B., physician, for twenty years has had eczema and for as long as he can remember has suffered at times from indigestion. The eczema began on scalp and those parts of the face covered with beard. The first attack extended to nearly every part of the surface of bedy. The eczematous patches of the surface were red and scaling, and occasionally moist. When he was a boy if he ate pickles, lemons, or other acid substances he suffered from heartburn, but of late vears the indigestion has been at times much more severe in character. When I examined the patient last autumn, I found that the scalp, neck, trunk. and popliteal spaces were the seats of the eczematous patches. A few of the diseased areas were moist, but most of them were dry and scaly. The patches on the trunk were of various sizes with well-defined borders and had all the objective signs of seborrheic eczema or seborrhea corporis, but the subjective symptoms were somewhat more severe than those which are generally present in cases of seborrheic eczema.

As the patient was suffering considerably from indigestion 1 gave him a test breakfast and analyzed the gastric contents. The total acidity and free $\mathrm{HCl}$ were 120 and 73 respectively; mucus slightly increased; digestion of starch very poor. The patient was therefore given an internal treatment suitable for hyperchlorhydria and an external treatment suitable for seborrheic eczema. We found that the lesions were very irrit- 
able. A mild resorcin and sulphur ointment, usually so effective in seborrheic ecrema, was not tolerated; but an ointment containing 8 grains of ammoniated mereury and 1 ounce of zinc oxid to an ounce of cold eream appeared to be soothing to the affected parts. Lnder this treatment a rapid improvement in the condition of the patient took place and two months later the rash had completely disappeared. I am of the opinion, therefore, that this was a case of seborrheic eczema aggravated by the irritable condition of the stomach.

CASE 3.-S. W., male, aged 27 , came to see me on June 10 , 1901. He told me that he had suffered from eczema of the face for over two years. On inquiring $I$ also ascertained that he frequently suffered from heartburn, pain after eating, belching and other symptoms of indigestion. His face and ears were nearly covered with red scaiy patches and his left cheek was considerably swollen. He also suffered from seborrhea of the scalp and alopecia furfuracea. I considered this a case of seborrheic eczema aggravated by hyperchlorhydria and prescribed accordingily. The rapid disappearance of the edema and the marked improrement in the scaly patches appear to support my diagnosis,

CASE 4.-A woman, aged 50, came to see me in December, 1900 , complaining of an eruption on the backs of her hands. She was full-blonded and had had her menopause about three years previously. She said that she had had indigestion for years, but the symptoms, discomfort after eating, belching, acid eructations, did not worry her very much as her appetite was fair and her general health was good. An examination of the lesions convinced me that it was a case of acute weeping eczema. and analysis of the gastric contents revealed the presence of hyperchlorhydria. The stomach was not displaced. I estimated the quantity of uric acid passed in a day to be $12 . \tilde{5}$ grains. I tried Garrod's test for uric acid in the blood and obtained a positive result.

The treatment of this patient was very similar to Case 1 . A mixture of black wash and calamin lotion was at first applied to hands. When the parts became dry I used Lassar's paste. Internally I gave an alkalin mixture and a light non-irritating diet.

CASE 5.-A. T., female, aged 40, came to my skin clinic at St. Michael's Hospital, June 5. She complained of an eruption on her thighs and face. She stated that the rash began on her thighs two years previously and about the same time she also began to suffer from indigestion-pain after eating, acid eructations, etc. Her digestion had improved of late, but the erup. tion was still on her thighs and had recently extended to her face.

An examination of the patient revealed the presence of scaly, eczematous patches on the thighs and an edematous erythematous eczema on the face. The blood was tested for uric acid by means of the thread-test and a marked deposit of uric acid crystals was obtained The patient was given a mixture of potassium bicarbonate, sodium salicylate, tincture of nux vomica and fluid extract of cascara sagrada aromatica before meals and a diet of bread, butter, milk and rice. In five days the eczema had completely disappeared from her face and in two weeks had nearly disappeared from her thighs. I then ordered a weak tar ointment, which in a few days effected a cure.

RELATIONS OF HYPERCHLORHYDRIA AND GOUT.

Disorders of the digestive system are of frequent occurrence in gout. All writers on the subject agree that excessive eating and drinking are important etiologic factors. They also agree that gout frequently gives rise to indigestion. "Acidity" is a common symptom in gouty subjects, and it has hitherto been held that the acid in the gastric contents was usually due to organic acids and not to hydrochloric acid. I believe that a thorough investigation of the subject would prove that this opinion is incorrect. We know that a similar erroneous idea was until recently held with regard to all cases of gastric indigestion. Deficiency and not excess of gastric secretion was said to be usually present in cases of dyspepsia. Even so distinguished a writer as Lauder Brunton, in his article in Clifford Allbutt's "System of Medicine," holds the same view. I know that this opinion is incorrect with regard to the dyspeptics in Toronto. During the last three years I must have examined the gastric contents of at least 300 patients and hyperchlorhydria was much more frequently present than hypochlorhydria.

The investigation of the relations of hyperchlorhydria to gout is somewhat difficult in this country, as according to my experience podagra is uncommon, while irregular gout is very common, but difficult to diagnose, particularly when not preceded by a history of gout in the foot. I have only examined the gastric contents of one patient with a history of regular gout, and he had marked hyperchlorhydria; but the subjective symptoms, referred to the stomach, which have been described to me by gouty patients: and which are generally held to be characteristic of the disease, are very similar to those of hyperchlorhydria. Again the etiologies resemble each other in some particulars. We know that excessive eating and daily use of alcoholic liquors in those who lead sedentary lives dispose to gout, and these are the same habits which are active agents in the production of hyperchlorhydria and hyperesthenic gastritis. It seems to me, therefore, that the relation between the two diseases is a subject worthy of investigation. If uratemia is shown to be present in cases of hyperchlorhydria then at least one important factor in the etiology of gout will have been determined.

\section{RELATION OF HYPERCHLORHYDRIA AND MOSCDLAR RHEUMATISM.}

We know very little about the etiology of muscular rheumatism. Exposure to cold is no doubt a contributing factor. Clinical experience teaches us that muscular rheumatism and gout are in some way related. It is probable, therefore, that patients with muscular rheumatism may suffer from a mild degree of uratemia.

In regard to relations of hyperchlorhydria and muscular rheumatism. I have observed that they are frequently associated, but whether the muscular rheumatism is the result of the hyperchlorhydria, I am at the present unable to say.

\section{URIC ACID INFLAMMATION OF THE MIDDLE EAR, MEMBRANA TYMPANI AND MASTOID.} CORNELIUS WILLIAMS, M.D. ST. PAUI, MINN.

I maintain that nine-tenths of all the inflammations of the ear and mastoid, as observed in the Northwest, are due to uric acid poisoning. In using this term, "uric acid poisoning," I mean to include its various compounds, and purely for convenience sake the several products of insufficient digestion as well as some results of faulty tissue metabolism.

Uric acid inflammation of the ear occurs in summer. and winter, but most frequently in late winter and early spring, and affects persons of all ages-babies in arms and the old as well. Those affected, according to my observation, have always been persons who ate nitrogenous food in excess of their requirements. The adults were rneat-eaters, often almost to the exclusion of vegetables, and the babies were bottle-fed. Further, there is generally a distinct history of gout or rheumatism, but more especially intestinal disorder due 